\title{
Characterizing the Utility and Limitations of Repurposing an Open-Field Optical Imaging Device for Fluorescence-Guided Surgery in Head and Neck Cancer Patients
}

\author{
Lindsay S. Moore ${ }^{1}$, Eben L. Rosenthal ${ }^{2}$, Thomas K. Chung ${ }^{1}$, Esther de Boer ${ }^{1,3}$, Neel Patel ${ }^{1}$, Andrew C. Prince ${ }^{1}$, \\ Melissa L. Korb ${ }^{4}$, Erika M. Walsh ${ }^{1}$, E. Scott Young ${ }^{5}$, Todd M. Stevens ${ }^{5}$, Kirk P. Withrow ${ }^{1}$, Anthony B. Morlandt ${ }^{6}$, \\ Joshua S. Richman ${ }^{4}$, William R. Carroll ${ }^{1}$, Kurt R. Zinn ${ }^{7}$, and Jason M. Warram ${ }^{1}$ \\ ${ }^{1}$ Department of Otolaryngology, University of Alabama at Birmingham, Birmingham, Alabama; ${ }^{2}$ Department of Otolaryngology, \\ Stanford University, Stanford, California; ${ }^{3}$ Department of Surgery, University of Groningen, Groningen, The Netherlands; \\ ${ }^{4}$ Department of Surgery, University of Alabama at Birmingham, Birmingham, Alabama; ${ }^{5}$ Department of Pathology, University of \\ Alabama at Birmingham, Birmingham, Alabama; ${ }^{6}$ Department of Oral and Maxillofacial Surgery, University of Alabama at \\ Birmingham, Birmingham, Alabama; and ${ }^{7}$ Department of Radiology, University of Alabama at Birmingham, Birmingham, Alabama
}

The purpose of this study was to assess the potential of U.S. Food and Drug Administration-cleared devices designed for indocyanine green-based perfusion imaging to identify cancer-specific bioconjugates with overlapping excitation and emission wavelengths. Recent clinical trials have demonstrated potential for fluorescenceguided surgery, but the time and cost of the approval process may impede clinical translation. To expedite this translation, we explored the feasibility of repurposing existing optical imaging devices for fluorescence-guided surgery. Methods: Consenting patients $(n=15)$ scheduled for curative resection were enrolled in a clinical trial evaluating the safety and specificity of cetuximabIRDye800 (NCT01987375). Open-field fluorescence imaging was performed preoperatively and during the surgical resection. Fluorescence intensity was quantified using integrated instrument software, and the tumor-to-background ratio characterized fluorescence contrast. Results: In the preoperative clinic, the open-field device demonstrated potential to guide preoperative mapping of tumor borders, optimize the day of surgery, and identify occult lesions. Intraoperatively, the device demonstrated robust potential to guide surgical resections, as all peak tumor-to-background ratios were greater than 2 (range, 2.2-14.1). Postresection wound bed fluorescence was significantly less than preresection tumor fluorescence $(P<0.001)$. The repurposed device also successfully identified positive margins. Conclusion: The open-field imaging device was successfully repurposed to distinguish cancer from normal tissue in the preoperative clinic and throughout surgical resection. This study illuminated the potential for existing open-field optical imaging devices with overlapping excitation and emission spectra to be used for fluorescence-guided surgery.

Key Words: fluorescence-guided surgery; optical imaging; head and neck cancer

J Nucl Med 2017; 58:246-251

DOI: 10.2967/jnumed.115.171413

Received Dec. 17, 2015; revision accepted Aug. 10, 2016.

For correspondence or reprints contact: Eben Rosenthal, Stanford Cancer Center, 300 Pasteur Dr, Stanford, CA 94305.

E-mail: elr@stanford.edu

Published online Sep. 1, 2016

COPYRIGHT @ 2017 by the Society of Nuclear Medicine and Molecular Imaging.
O treatment for many solid cancers. Unfortunately, close or positive surgical margins occur in $30 \%-40 \%$ of radical cancer resections (13 ) and have a direct impact on surgical outcomes (4-6). The intraoperative identification of tumor margins is inexact, as surgeons must rely on visual and manual identification of subtle tissue irregularities to discern tumor borders. Nonspecific anatomic imaging modalities can identify tumor boundaries with millimeter resolution and are being increasingly incorporated into the operating room; however, these modalities cannot provide real-time information and cannot easily be applied to the surgical field of view (7). Frozensection analysis provides guidance for intraoperative margin assessment but is time-intensive and can sample only a fraction of the wound bed (8). Optical imaging for cancer-specific surgical navigation offers a viable solution for real-time, intraoperative guidance of tumor resection and ex vivo margin analysis $(9,10)$. Fluorescence-guided surgery has been shown to increase complete resections and improve oncologic and functional outcomes in clinical trials using 5-aminolevulinic acid $(11,12)$. These results, in combination with the wealth of preclinical and clinical data supporting the benefits of fluorescence-guided surgery (13-20), have illuminated the need for clinical translation of this technique. As a result, more than 20 clinical trials using fluorescence-guided surgery during surgical oncology were currently ongoing in 2015 according to clinicaltrials.gov (7), thus highlighting the potential application of this technology in the surgical management of cancer.

Fluorescence-guided surgery requires a fluorescent probe and a compatible imaging device. Clinical translation of these components has been limited because the development and approval of standardized imaging devices and agents is expensive, of unknown toxicity, and time-intensive, with limited expected return compared with traditional therapeutic agents (7). There is a critical need for industry to develop this technology in order to create a clinical infrastructure for routine use. However, stiff regulatory requirements and an unknown reimbursement status may deter industry from investing in a pathway aimed at designing and marketing an intraoperative imaging device specifically optimized for surgical oncology.

One option is to pair the fluorescent agent and the device so that they are required to be used together, as is currently done for 
optical agents $(7,21,22)$. An alternative approach to streamline the clinical implementation of fluorescence surgical navigation is to allow new or repurposed devices to be used with several fluorescent agents that have excitation and emission spectra falling within the range of the device parameters. Thus, one device could be used for several fluorescent agents and one agent could be used with multiple devices. This strategy is not without its own unique set of challenges, including the use of a fluorophore that is not optimal for the spectrum of the device and, in the case of repurposed devices, limited intraoperative applications due to size, ergonomics, or integrated software constraints (7). However, repurposing a preapproved device ensures patient safety (as the device's safety profile is already known) and is substantially more time-, cost-, and resourceeffective (7). Repurposing existing optical imaging devices for use in fluorescence-guided surgery may therefore provide the most straightforward and efficient path to the clinic.

The objective of this study was to characterize the value and limitations of using an approved intraoperative open-field, nearinfrared (NIR) optical imaging system (LUNA; Novadaq) for navigation during oncologic surgical resections. Furthermore, we sought to determine whether a device designed around one fluorophore could be used to imaging another NIR dye, IRDye800CW (Li-COR Biosciences), with an overlapping emission and excitation spectra. This investigation was performed in the context of a clinical trial (Clinicaltrials.gov trial NCT01987375) evaluating the safety and specificity of cetuximab-IRDye $800 \mathrm{CW}$ in patients with head and neck cancer (23).

\section{MATERIALS AND METHODS}

\section{Study Design}

The LUNA system was repurposed to fluorescently localize cetuximabIRDye $800 \mathrm{CW}$ in 15 patients scheduled for a curative surgical resection of squamous cell carcinoma of the head and neck. Consenting patients were enrolled in a dose-escalating study evaluating the safety and specificity of cetuximab conjugated to IRDye800CW (excitation, 774 $\mathrm{nm}$; emission, $789 \mathrm{~nm}$ ), as previously described (23). The escalating doses were based on the therapeutic dose of cetuximab $\left(250 \mathrm{mg} / \mathrm{m}^{2}\right)$. Cohort 1 ( 3 patients) was given a microdose $(1 \%$ of the therapeutic dose), cohort 2 (9 patients) received $10 \%$ of the therapeutic dose, and cohort 3 (3 patients) received $25 \%$ of the therapeutic dose (23). All patients gave written informed consent, and the Institutional Review Board approved the study protocol and the process by which the University of Alabama at Birmingham Vector Production Facility manufactured the cetuximab-IRDye800CW (24).

\section{Optical Imaging}

The LUNA system has a laser excitation waveband at $805 \mathrm{~nm}$ and an imaging long-pass filter that collects NIR emission wavelengths greater than $820 \mathrm{~nm}$. Supplemental Figure 1A illustrates the overlap between the absorption and emission spectra of IRDye800CW and the optics range of the LUNA system (supplemental materials are available at http://jnm.snmjournals.org). A standardized comparison of the LUNA system and a closed-field device optimized to image IRDye 800CW is shown in Supplemental Figure 1B, and a comparison of the tumor-to-background ratios (TBRs) for the same specimen taken by each device for 3 representative patients is shown in Supplemental Figure 1C. Open-field imaging of the primary tumor site, ipsilateral neck, forearm, and oral mucosa was performed in the preoperative clinic $3 \mathrm{~h}$ after injection of cetuximab-IRDye800CW (day 0 ), every $24 \mathrm{~h}$ thereafter (per patient availability), and before resection on the day of surgery (day 3-7 after infusion) (Supplemental Fig. 2A). Real-time, open-field imaging of the primary specimen and postresec- tion wound bed was performed intraoperatively in the surgical field (Supplemental Fig. 2B). Resected primary specimens and resected margins were imaged ex vivo with the LUNA system immediately after resection in the operating room (Supplemental Fig. 2C). This intraoperative approach, termed ex vivo fluorescence imaging for neoplastic detection (xFIND), involves NIR fluorescence imaging of specimens on a table within the operating room immediately after excision, before frozen sectioning and pathologic processing. xFIND is designed to provide rapid, real-time information about the presence and location of cancer-containing tissue to guide resection without interrupting the surgical workflow. Image settings were constant throughout acquisition, with the camera at a distance of 30 or $15 \mathrm{~cm}$.

\section{Fluorescence Analysis}

Fluorescence was quantified using integrated instrument software (SpyQ, version 1.2; Novadaq). Relative fluorescence units (RFU) were measured for tumor and background $(\sim 3-4 \mathrm{~cm}$ from the edge of the gross tumor) and averaged among 6 individual frames per imaging time point. The postresection wound bed was also imaged in these same regions as the primary tumor for each patient. TBR was calculated by dividing tumor RFU by respective background RFU as previously described (25). For qualitative analysis, exported DICOM files were used to produce videos and images using OsiriX (26) with standardized thresholds.

\section{Histologic Assessment}

After routine hematoxylin and eosin staining, histologic assessment was performed by a board-certified pathologist. The stained slides were imaged using the BioImagene (Ventana Medical Systems) optical scanner. The Odyssey imaging platform (LI-COR Biosciences) was used to identify fluorescence in slide-mounted sections obtained from paraffin-embedded blocks.

\section{RESULTS}

\section{Preoperative Imaging}

Open-field NIR imaging with the LUNA system was performed in the clinic on the day of cetuximab-IRDye $800 \mathrm{CW}$ infusion and daily thereafter, including before resection on the day of surgery. As shown for a representative patient in Figure 1A, the open-field device successfully demonstrated robust potential for preoperative surface mapping of superficial tumor borders and initial incision sites using the fluorescence border and the optimal 5-mm resection margin for cutaneous squamous cell carcinomas. Additionally, analysis of tumor and background intensity during daily preoperative clinical imaging revealed peak fluorescence contrast as determined from TBR values (Fig. 1B). Quantitative assessment confirmed that the average RFU of the primary tumor in situ $(95.8 \pm 2.04)$ and the average TBR $(2.78 \pm 0.04)$ were greatest on day 1 after dye infusion. This is also shown qualitatively in Figure 1B with inset bright-field and fluorescence images acquired at each time point.

In another case, shown in Figure 1C, a patient presented with a locoregional, recurrent oral cavity lesion, and a small (5-mm) lesion was noted in the scar of a previous neck dissection. The occult lesion was first identified by in situ fluorescence imaging in the preoperative clinic, prompting additional inspection and subsequent surgical excision at the time the primary tumor was resected. Ex vivo fluorescence imaging of the excised specimen revealed greater levels of fluorescence at the site of the lesion (Fig. 1C). The presence of cancer in the areas of high fluorescence intensity was confirmed by histopathology. 




FIGURE 1. Utility of LUNA system in preoperative clinic. (A-C) Device can be used for presurgical mapping of tumor margins (A), daily imaging to determine optimal day of surgery (B), and detection and evaluation of occult or suggestive superficial lesions (C). Dotted line in A depicts fluorescence margin, and dashed line denotes $5-\mathrm{mm}$ resection margin. Fluorescence images in $B$ are shown with representative bright-field images taken on days 0 (left) and 7 (right). Arrows in $\mathrm{C}$ denote pathologyproven malignant cutaneous metastasis in situ and ex vivo.

\section{Operating Room}

Intraoperative fluorescence imaging of the primary tumor was performed before, during, and immediately after surgical resection to assess the feasibility of repurposing the open-field NIR imaging device to collect valuable, real-time imaging data suitable for fluorescence-guided surgery. In general, the LUNA system was easily and unobtrusively incorporated into the surgical workflow (Supplemental Fig. 2B). Imaging conducted before and throughout

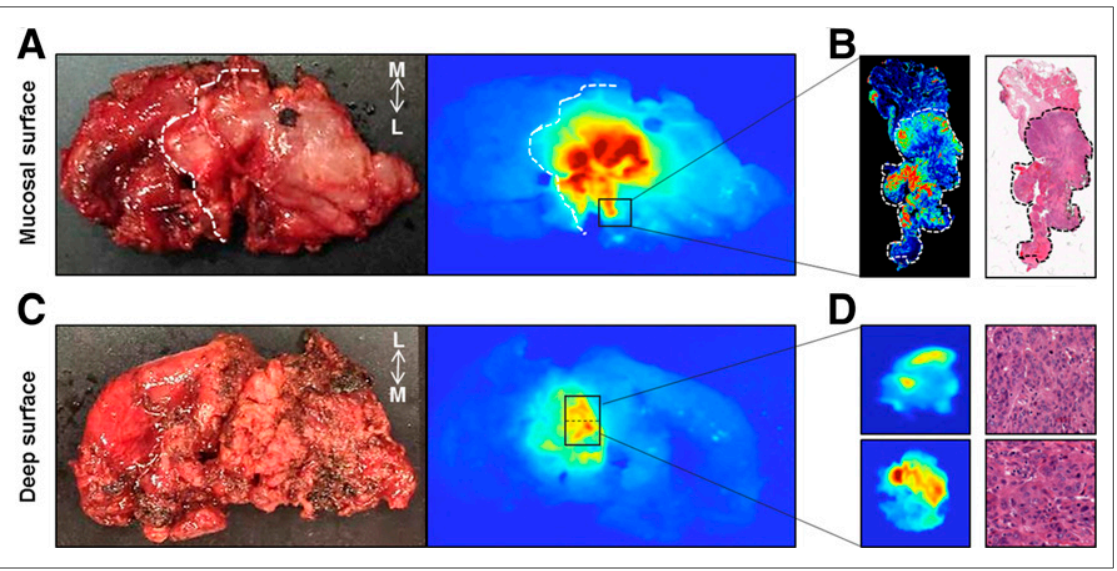

FIGURE 2. EX vivo imaging of resected primary piriform recess tumor and margins. (A and B) Bright-field and corresponding fluorescence images of mucosal surface $(A)$ and deep surface (C) of resected primary specimen are shown. Dashed line denotes cut mucosal border. (B) Histology of positive lateral mucosal margin is shown with fluorescence histology and hematoxylin and eosin staining. Dashed lines denote cancerous areas. (D) Fluorescence images of lateral (top) and medial (bottom) margins taken from wound bed after fluorescence imaging of deep surface demonstrated positive deep margin, and histopathology confirmed presence of cancer in each of these margins. $L=$ lateral; $M=$ medial. the resection demonstrated robust contrast between the tumor and surrounding normal tissue in all patients except those in the microdose cohort (cohort 1). Quantitatively, imaging of the primary tumor in situ yielded fluorescence contrast with a peak TBR greater than 2 (range, 2.2-14.1) for all patients in cohorts 2 and 3 , and there was no significant difference in TBRs between these two cohorts. Supplemental Figure 3A shows tumor (preresection) fluorescence to be significantly $(P<0.001)$ higher than patient-matched background fluorescence in 3 representative patients.

After resection of the primary tumor, open-field imaging was used to assess the utility of the repurposed device to survey the postresection wound bed for residual cancerous tissue. Preresection tumor, preoperative adjacent background, and postresection wound bed fluorescence values for 3 representative patients are quantified in Supplemental Figure 3A. During the analysis, the preresection fluorescence intensity of the primary tumor in situ $(20.6 \pm 4.6)$ was shown to be significantly $(P<0.001)$ greater than both background fluorescence $(6.3 \pm 0.5)$ and postresection wound bed fluorescence $(2.0 \pm 0.8)$. Supplemental Figure $3 \mathrm{~B}$ shows representative bright-field and fluorescence images of the underwent total glossectomy.

The open-field device was also used during ex vivo imaging of the resected primary specimen and margins to explore the device's potential for xFIND imaging, designed to provide a real-time assessment of surgical margins within the operating room. Bright-field and fluorescence images of an ex vivo piriform recess tumor showed areas of strong fluorescence on both the mucosal surface (Figs. 2A and 2B) and the deep surface (Figs. 2C and 2D) during xFIND imaging with the open-field device. On the mucosal surface, the area of high fluorescence at the lateral margin of the resected primary specimen (Fig. 2A) correlated with the closest pathologically positive margin. Imaging was confirmed by fluorescence acquisition (Odyssey) of the slide-mounted pathologic section with a closed-field flatbed scanner optimized for imaging IRDye800 and the corresponding hematoxylin and eosin section (Fig. 2B), which shows annotated tumor areas as determined by a boardcertified pathologist using hematoxylin and eosin analysis. On the deep surface of the resected primary specimen, openfield fluorescence imaging (Figs. 2C and 2D) successfully identified a histologyproven positive deep margin. The two margins shown in Figure 2D were subsequently resected from the area of the wound bed corresponding to the positive deep margin and were xFIND-imaged with the open-field device. Regions of high fluorescence correlated with the fluorescently positive deep margin of the primary as mapped in Figures 2C and $2 \mathrm{D}$ and were confirmed to contain cancer by a board-certified pathologist using hematoxylin and eosin staining (Fig. 2D).

\section{Limitations of Imaging}

Figure 3 illustrates the limitations of fluorescence imaging in the oral cavity. In 




FIGURE 3. In situ imaging of oral cavity lesions is limited. (A-C) Brightfield and corresponding fluorescence images of oral cavity lesions in 3 representative patients demonstrate difference in fluorescence between in situ and ex vivo imaging. Arrows denote cancerous areas. (D) Quantitative analysis demonstrates increase in TBR of oral cavity lesions from in situ to ex vivo imaging for all 3 patients.

all 3 patients with oral cavity cancer (hard palate and lateral tongue), the fluorescence intensity observed for each lesion when imaged in situ was significantly less (87\% reduction) than the fluorescence intensity of the same lesion when imaged ex vivo $(P<0.05$ for all). This difference is shown qualitatively in Figure $3 \mathrm{~A}-3 \mathrm{C}$ when the in situ and ex vivo fluorescence images of each patient's lesion are compared (shown with corresponding brightfield images). Additionally, quantitative analysis in Figure 3D demonstrates the reduced fluorescence contrast (TBR) of in situ

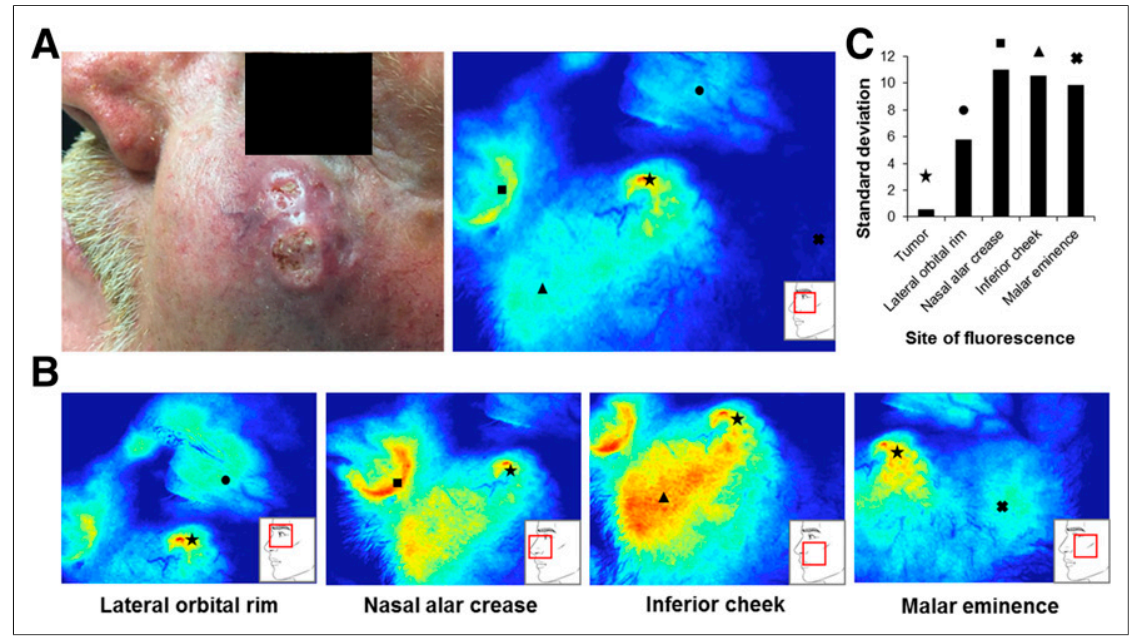

FIGURE 4. Dynamic fluorescence imaging of cutaneous squamous cell carcinoma. (A) Bright-field and corresponding fluorescence image of lesion when device was orthogonal to primary lesion $(\star)$. (B) Images of same lesion when camera was focused on surrounding areas of fluorescence artifact: lateral orbital $\operatorname{rim}(\bullet)$, nasal alar crease $(\boldsymbol{\bullet})$, inferior cheek $(\mathbf{\Lambda})$, and malar eminence $(\mathbf{X})$. Insets at bottom right denote imaging field at each camera position. (C) Fluorescence of tumor and 4 subsites was measured at each camera position, and SD for each site between frames is shown. imaging compared with ex vivo imaging of oral cavity cancer in each of the patients.

When we were imaging the complex topography of head and neck anatomy with the open-field device, off-target areas of elevated fluorescence intensity were frequently encountered in healthy tissues and structures. Dynamic fluorescence imaging of a patient with cutaneous squamous cell carcinoma of the lateral cheek (Fig. 4A) identified 4 surrounding regions exhibiting elevated levels of fluorescence although appearing noncancerous on gross examination. Each of the fluorescence images (Fig. 4B) was acquired with the device's camera oriented to capture a slightly different perspective, containing the primary lesion and 1 of the 4 noncancerous subsites: lateral orbital rim, nasal alar crease, inferior cheek, and malar eminence. The fluorescence intensity of each subsite fluctuated and was dependent on the perspective, whereas-importantly - the fluorescence of the primary lesion remained nearly constant regardless of the perspective. This finding is quantified in Figure 4C, which shows the SD of the mean RFU for the tumor (0.55 RFU) and the subsites (5.7410.5 RFU). The SD was 10 - to 20 -fold lower for the tumor than for any of the subsites.

\section{DISCUSSION}

This study established the feasibility and utility of repurposing the most current commercially available model of an openfield imaging device intended for fluorescence angiography to provide real-time information to guide surgical resection of solid tumors. In the preoperative clinic, the potential of the device to guide preoperative mapping, identify occult disease, and identify the optimal day of surgical resection was shown. In the operating room, the repurposed device demonstrated potential to guide surgical resection of tumors in real time and localize disease in the wound bed, the excised primary specimen, and the margins. Given the breadth of utility and the potential to provide valuable, real-time information before, during, and after surgical resection, repurposing an open-field fluorescence imaging device can provide a valuable adjunct to the oncologic surgeon.

Although most imaging devices are not paired with contrast agents, experimental optical agents are currently paired with a corresponding imaging device. As such, exploring the feasibility of using a single device, especially those that are already commercially available, to image multiple probes is of current interest to many investigators in the field. Schols et al. applied a commercially available NIR laparoscopic imaging device designed for use with indocyanine green to image biliary anatomy using IRDye $800 \mathrm{CW}$ in pigs (27). We have demonstrated that the limited wavelength overlap was not a deterrent to successful tumor identification or delineation as confirmed by histology. In fact, the familiarity of the operating room staff and surgeon with the device from prior experience with it significantly reduced operative time and improved interpretation 
of the images. This finding has regulatory implications for the pairing of devices with fluorophores.

However, with the advent of high-power NIR laser diodes and tunable filters, future devices may be interchangeable and amenable to the excitation spectrum of the selected NIR probe. Additionally, because indocyanine green is dosed in relatively large $(\mathrm{mg})$ quantities for fluorescence angiography, the fluorescence emission detection parameters of indocyanine green imaging systems are not optimal for detecting the very low optical emission that is required to detect microscopic, subclinical disease. To overcome this problem, software and image capture modifications may be incorporated to provide the sensitivity needed to detect small islands of cancer and permit localization of disease in the unique intraoperative setting of surgical resection.

The mobility and ergonomic design of the open-field device allows for the use of a single device in both the operating room and the preoperative clinic. Additional studies will be needed to determine whether fluorescence-enhanced preoperative mapping can be used to ensure that the primary specimen is resected in its entirety and whether tissues may be spared by limiting overresectioning. The preoperative utility was also shown to be valuable in assessing tumor fluorescence to identify peak contrast and the optimal day for the surgical resection. Additionally, the open-field device was able to detect cancer in a suggestive lesion during regional assessment, illuminating the potential of the device to provide rapid, real-time assessment of a region of anatomy, or even the whole body, to detect occult lesions or quickly assess suggestive ones.

Although this study illuminated an impressive number of uses for the open-field device in both the preoperative clinic and the operating room, the few limitations encountered in this study offer equally valuable insights into the future of open-field devices designed specifically for surgical navigation of oncologic resections. First, because the open-field device in our study was designed for cutaneous and surface imaging, the ergonomic form of the camera-head limited imaging of small cavities. The angle of the excitation source relative to the charge-coupled-device camera lens in this device is designed for fluorescence angiography and directly interferes with cancer imaging, especially in small cavities. In view of this limitation, it is envisioned that an openfield device designed specifically for cancer resection may require a handheld or even endoscopic probe to permit evaluation of complex 3-dimensional ablative defects in real time. Additionally, approximation of the excitation source and the charge-coupleddevice camera lens, perhaps with the light source positioned in ring configuration around the camera lens, may significantly improve fluorescence cancer imaging.

Another limitation observed during the study was inherent in the nature of optical imaging; the fluorescent signal varies as a function of distance. When anatomy with complex topography and varied geography is imaged, the surfaces in a given frame that are closest and most orthogonal to the camera will appear relatively more fluorescent. Additionally, in areas at which two planes of tissue converge on a single point, such as the nasal alar crease, the camera aperture may perceive additive fluorescence. Although this artifact may confound analysis of still acquisitions, dynamic imaging was shown to easily distinguish areas of fluorescence artifact, which change relative to the camera orientation, from areas of tumor-specific fluorescence, which remain relatively bright regardless of camera orientation. It is expected that surgeons using this technology would easily develop the pattern-recognition skills required to distinguish true signal from artifact. However, as Figure 4C demonstrates, analysis of the differences in SD for an area between two camera perspectives can be used to develop an algorithm for automated determination of true-positive areas.

\section{CONCLUSION}

This study illuminated the potential for repurposed intraoperative open-field imaging devices not specifically paired with an imaging agent to guide the surgical management of solid tumors. This study also suggested that devices may not need to be paired with fluorescent probes optimized for a specific range of excitation and emission spectra. This possibility would provide for a time- and cost-effective route to clinical translation of this promising field.

\section{DISCLOSURE}

This work was funded by the Robert Armstrong Research Acceleration Fund; the University of Alabama at Birmingham Comprehensive Cancer Center; grants R21CA179171, R21CA182953, T32CA091078 from the NIH/NCI; and institutional equipment loans from Novadaq and LI-COR Biosciences. No other potential conflict of interest relevant to this article was reported.

\section{REFERENCES}

1. Woolgar JA, Triantafyllou A. A histopathological appraisal of surgical margins in oral and oropharyngeal cancer resection specimens. Oral Oncol. 2005;41:1034 1043.

2. McMahon J, O'Brien CJ, Pathak I, et al. Influence of condition of surgical margins on local recurrence and disease specific survival in oral and oropharyngeal cancer. Br J Oral Maxillofac Surg. 2003;41:224-231.

3. Ravasz LA, Slootweg PJ, Hordijk GJ, et al. The status of the resection margin as a prognostic factor in the treatment of head and neck carcinoma. J Craniomaxillofac Surg. 1991;19:314-318.

4. Hinni ML, Ferlito A, Brandwein-Gensler MS, et al. Surgical margins in head and neck cancer: a contemporary review. Head Neck. 2013;35:1362-1370.

5. Cook JA, Jones AS, Phillips DE, et al. Implications of tumour in resection margins following surgical treatment of squamous cell carcinoma of the head and neck. Clin Otolaryngol. 1993;18:37-41.

6. Byers RM, Bland KI, Borlase B, et al. The prognostic and therapeutic value of frozen section determinations in the surgical treatment of squamous carcinoma of the head and neck. Am J Surg. 1978;136:525-528.

7. Rosenthal EL, Warram JM, deBoer E, et al. Successful translation of fluorescence surgical navigation during oncologic surgery: a consensus report. $\mathrm{J} \mathrm{Nucl}$ Med. 2016;57:144-150.

8. Twaalfhoven FCM, Peters AAW, Trimbos JB, et al. The accuracy of frozen section diagnosis of ovarian tumors. Gynecol Oncol. 1991;41:189-192.

9. Rosenthal EL, Warram JM, Bland KI, et al. The status of contemporary imageguided modalities in oncologic surgery. Ann Surg. 2015;261:45-55.

10. Keereweer S, Van Driel PBAA, Snoeks TJA, et al. Optical image-guided cancer surgery: challenges and limitations. Clin Cancer Res. 2013;19:37453754 .

11. Stummer W, Novotny A, Stepp H, et al. Fluorescence-guided resection of glioblastoma multiforme by using 5-aminolevulinic acid-induced porphyrins: a prospective study in 52 consecutive patients. J Neurosurg. 2000;93:10031013.

12. Stummer W, Pichlmeier U, Meinel T, et al. Fluorescence-guided surgery with 5aminolevulinic acid for resection of malignant glioma: a randomised controlled multicentre phase III trial. Lancet Oncol. 2006;7:392-401.

13. Day KE, Sweeny L, Kulbersh B, et al. Preclinical comparison of near-infraredlabeled cetuximab and panitumumab for optical imaging of head and neck squamous cell carcinoma. Mol Imaging Biol. 2013;15:722-729. 
14. Korb ML, Hartman YE, Kovar J, et al. Use of monoclonal antibody-IRDye800CW bioconjugates in the resection of breast cancer. J Surg Res. 2014; 188:119-128.

15. Day KE, Beck LN, Deep NL, et al. Fluorescently labeled therapeutic antibodies for detection of microscopic melanoma. Laryngoscope. 2013;123:26812689.

16. Rosenthal EL, Kulbersh BD, Duncan RD, et al. In vivo detection of head and neck cancer orthotopic xenografts by immunofluorescence. Laryngoscope. 2006; 116:1636-1641.

17. Kulbersh BD, Duncan RD, Magnuson JS, et al. Sensitivity and specificity of fluorescent immunoguided neoplasm detection in head and neck cancer xenografts. Arch Otolaryngol Head Neck Surg. 2007;133:511-515.

18. van der Vorst JR, Schaafsma BE, Verbeek FP, et al. Near-infrared fluorescence sentinel lymph node mapping of the oral cavity in head and neck cancer patients. Oral Oncol. 2013;49:15-19.

19. Tanaka E, Choi HS, Humblet V, et al. Real-time intraoperative assessment of the extrahepatic bile ducts in rats and pigs using invisible near-infrared fluorescent light. Surgery. 2008;144:39-48.

20. Tanaka E, Ohnishi S, Laurence RG, et al. Real-time intraoperative ureteral guidance using invisible near-infrared fluorescence. J Urol. 2007;178:2197-2202.
21. Premarket approval (PMA). U.S. Food and Drug Administration website. http:// www.accessdata.fda.gov/scripts/cdrh/cfdocs/cfPMA/pma.cfm. Updated September 26, 2016. Accessed September 27, 2016.

22. $510(\mathrm{k})$ premarket notification. U.S. Food and Drug Administration website. http://www.accessdata.fda.gov/scripts/cdrh/cfdocs/cfpmn/pmnsimplesearch.cfm. Updated September 26, 2016. Accessed September 27, 2016.

23. Rosenthal EL, Warram JM, de Boer E, et al. Safety and tumor specificity of cetuximab-IRDye 800 for surgical navigation in head and neck cancer. Clin Cancer Res. 2015;21:3658-3666.

24. Zinn KR, Korb M, Samuel S, et al. IND-directed safety and biodistribution study of intravenously injected cetuximab-IRDye800 in cynomolgus macaques. Mol Imaging Biol. 2015;17:49-57.

25. Heath $\mathrm{CH}$, Deep NL, Sweeny L, et al. Use of panitumumab-IRDye800 to image microscopic head and neck cancer in an orthotopic surgical model. Ann Surg Oncol. 2012;19:3879-3887.

26. Rosset A, Spadola L, Ratib O. OsiriX: an open-source software for navigating in multidimensional DICOM images. J Digit Imaging. 2004;17:205-216.

27. Schols RM, Lodewick TM, Bouvy ND, et al. Near-infrared fluorescence laparoscopy of the cystic duct and artery in pigs: performance of a preclinical dye. $J$ Laparoendosc Adv Surg Tech A. 2014;24:318-322. 